\title{
Is endocervical curettage necessary? A literature review
}

\author{
Karen Herold \\ California State University, Los Angeles, United States \\ Correspondence: Karen Herold. Address: California State University, Los Angeles, United States. Email: \\ kherold@csula.edu
}

Received: November 10, 2011

Accepted: January 3, $2012 \quad$ Published: May 1, 2012

DOI : $10.5430 /$ jnep.v2n2p98

URL: http://dx.doi.org/10.5430/jnep.v2n2p98

\section{Abstract}

Background: Invasive cervical cancer develops from a pre-invasive state named cervical intraepithelial neoplasia (CIN). CIN 1 represents mild dysplasia and is now classified as low-grade squamous intraepithelial lesion (LGSIL); CIN 2 and 3 lesions encompass moderate-to-severe dysplasia and are now classified as high-grade squamous intraepithelial lesions (HGSILs) based on the Bethesda cervical cytology reporting system. Most LGSIL lesions resolve spontaneously, whereas HGSIL lesions are more likely to progress to invasive cervical cancer. However, HGSILs are typically detected an average of 10 to 15 years before invasive cervical cancer. In women, the typical age range for diagnosis of invasive cancer is greater than 40 years. High-risk Human Papilloma Virus (HRHPV) infection is required for the development of virtually all cervical cancer, and the time from initial HPV infection to development of cervical cancer usually exceeds 10 years. Colposcopies that are performed for abnormal papanicolaou (pap) smears of LGSIL and HRHPV can include ectocervical and endocervical biopsies. Given the likelihood of (a) spontaneous resolution of LGSIL and HRHPV, (b) the time to development of cervical cancer after initial diagnosis of HPV and (c) the improbability of invasive cervical cancer developing in women less than 40 years of age, there is evidence to suggest that endocervical curettage at the time of colposcopy is not necessary in a subgroup of women who are at low risk of developing invasive cervical cancer. At the current time, no evidence based guidelines exist about the necessity of endocervical curettage (ECC) at the time of colposcopy in women who are at low risk for invasive cervical cancer. Therefore, a literature review was conducted to analyze literature as it related to ECC at the time of colposcopy in who are at low risk for invasive cervical cancer. A particular subgroup of women that are at low risk for development of cervical cancer are less than 35 years of age with LGSIL or HRHPV cytology, and women who are less than 35 years of age are at an even lower risk of developing invasive cervical cancer because of their more robust immune system. The purpose of the literature review was to summarize findings about endocervical curettage at the time of colposcopy to propose a study that could be conducted to establish evidence based guidelines for ECC that could be utilized in practice.

Methods: The literature review summarizes current empirical and theoretical knowledge related to ECC at the time of colposcopy in women who are less than 35 years of age with LGSIL or HRHPV cytology. Concepts and keywords of the proposed literature review include: ECC-based diagnosis in each of three categories (benign, CIN 1 and CIN $>1$ ), ectocervical-based biopsy in each of three categories (benign, CIN 1 and CIN >1), LGSIL, HRHPV, colposcopy, and cervical cancer. Primary and secondary literature was examined to elucidate gaps in the literature related to evidence based guidelines for ECC at the time of colposcopy. 
Results: The literature review includes an analysis of the literature related to ECC at the time of colposcopy in women who were less than 35 years of age with Cervical Cytology of LGSIL or HRHPV. Additionally, the literature review provides a critique of the studies that were reviewed, details gaps in the literature and enumerates the problem of no evidence based guidelines for ECC curettage at the time of colposcopy.

Conclusions: There is an absence in the literature about evidence based guidelines for performing ECC at the time of colposcopy in women who are less than 35 years of age with LGSIL or HRHPV; and additionally a paucity exists in the literature about ECC at the time of colposcopy in this same subgroup of women. Therefore, the literature review establishes the foundation and background for future studies that examine the need for ECC in Women less than 35 years of age with Cervical Cytology of LGSIL or HRHPV at the time of colposcopy.

\section{Key words}

Colposcopy, Low-Grade Intraepithelial Lesion (LGSIL), High Risk Human Papilloma Virus (HRHPV), Endocervical curettage, Ectocervical biopsy, Cervical cancer

\section{I ntroduction}

The primary goal of a literature review is to objectively analyze a published body of knowledge about previous research that has been conducted on a specific topic by recognized researchers. A completed Literature review is a summary, categorization and comparison of previous research studies and may suggest areas for further research. Additionally a literature review informs the reader about the current concepts of research on a specific topic, and may identify gaps or lacks in the literature related to the topic.

The use of ECC has been debated in the literature by researchers over the past 40 years ${ }^{[1]}$. Currently, there are no evidence based guidelines for the performance of ECC at the time of colposcopy for women who are less than 35 years of age with low-grade cervical cytology. A literature review was conducted to summarize findings related to ECC at the time of colposcopy and to examine existing literature about endocervical curettage ECC at the time of colposcopy. The literature was then analyzed to formulate a research question that could be used to conduct a retrospective study.

\section{Methods}

According to Auston, Cahn and Selden ${ }^{[2]}$ a literature search is a systematic and explicit approach to the identification, retrieval, and bibliographical management of independent studies for the purpose of locating information on a topic, synthesizing conclusions, identifying areas for future study, and developing guidelines for clinical practice. The goal of the literature review was to retrieve as many journal articles as possible about ECC and Colposcopy in a low risk population of women within a reasonable period of time; and to summarize current empirical and theoretical knowledge about this topic. Primary and secondary literature was examined to elucidate gaps in the literature related to evidence based guidelines for ECC at the time of colposcopy. Empirical literature was researched utilizing the following search engines: Index Medicus, Cochrane Library, PubMed, and Cinahl.

The initial search of the literature was based on the widest interpretation of the literature as was possible related to ECC and Colposcopy in a low risk population of women. Therefore a broad range of topics and keywords to examine the literature was utilized and included: (a) false positive or false negative ECC results, (b) contamination of ECC specimens, (c) pathology of endocervical canal no deeper than colposcopy, (d) effectiveness of ECCs depending on grade of lesion, (e) opposition to ECC, (f) argument for ECC at time of colposcopy, (g) using ECCs as predictors for micro invasion, (h) the need for ECC with cytology of CIN 1, and (i) necessity of ECCs in women less than 35 years of age, (j) colposcopy, (k) Low-Grade Intraepithelial Lesion (LGSIL), (1) High Risk Human Papilloma Virus (HRHPV), (m) Endocervical biopsy, (n) ectocervical biopsy, (o) cervical cancer, (p) Colposcopy, (q) ECC, ( r) ectocervical biopsy, (s) infection with HRHPV 
and time to progression of cervical cancer, $(\mathrm{t})$ women less than 35 years of age with cervical cytology, $(\mathrm{u})$ women less than 35 years of age with abnormal pap smears, (v) women less than 35 years of age with LGSIL, (w) women less than 35 years of age with HRHPV, (x) LGSIL and HRHPV and rate and time to spontaneous regression, (y) the time of progression of cervical cancer to invasive cervical cancer, (z) evidence based guidelines for colposcopy, and (aa) evidence based guidelines for ECC. The literature review summarizes current empirical and theoretical knowledge related to ECC at the time of colposcopy in women who were less than 35 years of age with Cervical cytology of LGSIL or HRHPV. Primary and secondary literature was examined to elucidate gaps in the literature related to evidence based guidelines for ECC at the time of colposcopy. Empirical literature was researched utilizing the following search engines: Index Medicus, Cochrane Library, PubMed, and Cinahl.

To scientifically monitor the process of gathering information for the literature review, it was important to systematically make a note about the sources that were utilized for retrieving the literature. To achieve this, a table was created to organize the literature and is presented in Table 1. Organization of the literature was accomplished by categorizing articles by title, author and publication and then further categorizing each articles purpose, variables, subjects, and data. Comments were made in the last section of the matrix regarding each retrieved article to establish relevance and importance.

Table 1. Matrix for Literature Review

\begin{tabular}{|c|c|c|c|c|c|c|c|c|c|c|}
\hline \multirow{2}{*}{$\begin{array}{l}\text { Authors, } \\
\text { Title, } \\
\text { Journal }\end{array}$} & \multirow[b]{2}{*}{$\begin{array}{l}\text { Year } \\
\text { Pub. }\end{array}$} & \multirow[b]{2}{*}{ Purpose } & \multicolumn{2}{|c|}{ Variables } & \multicolumn{3}{|l|}{ Subjects } & \multicolumn{2}{|l|}{ Data } & \multirow[b]{2}{*}{ Comments } \\
\hline & & & Dep. & Indep. & $\#$ of sub. & $\begin{array}{l}\text { Charac- } \\
\text { teristics }\end{array}$ & $\begin{array}{l}\text { Sample } \\
\text { design }\end{array}$ & $\begin{array}{l}\text { Source } \\
\text { or Instrument }\end{array}$ & $\begin{array}{l}\text { Yr. data } \\
\text { collected }\end{array}$ & \\
\hline & & & & & & & & & & \\
\hline & & & & & & & & & & \\
\hline
\end{tabular}

\section{Results}

The literature review resulted in the construction of concepts that were classified into the following subheadings: no lesions deeper than indicated by colposcopy, ECC depending on grade of lesion or dysplasia, opponents of ECC, false positive results and false negative, contamination, argument for doing ECC because of potential for missing serious lesions (and/or the ECC as the only manifestation of cervical disease), using ECC as a predictive value for micro invasion, is ECC necessary in patients with CIN 1 and adequate colposcopic examination?, is it worth performing ECCs in women less than 35 years old?, marginal diagnostic yield, diagnostic utility and gaps in the literature related to ECC.

\section{No lesions deeper than indicated by colposcopy}

Krebs et. al. ${ }^{[3]}$ identified a higher rate of cancer diagnosed in women who had an unsatisfactory colposcopy (9\% vs. $1.5 \%$ ), but even with a $5 \%$ positive ECC rate in women who had a satisfactory colposcopy, no lesions were deeper in the endocervical canal than predicted by colposcopy. Therefore, ECC was considered unnecessary in women who had a satisfactory colposcopic examination. From this study, researchers established the fact that in an adequate colposcopy the ECC gives no more additional useful information than the ectocervical biopsy.

\section{ECC depending on grade of lesion or dysplasia}

Depending on the grade of referral cytology researchers differed in their argument for or against colposcopy. Examples of this included: Saltzman et al. ${ }^{[4]}$ noted that ECC was positive in $260 \%$ more cases in which cervical intraepithelial 
neoplasia (CIN 3) was present than those of CIN 1 or 2. This study indicated that ECC may not be necessary in patients with HRHPV or LGSIL.

Williams et al. ${ }^{[5]}$ studied women who had atypical squamous cells of undetermined significance (ASCUS), LGSIL, and normal colposcopy. In this study, the ECC was positive in only $2.5 \%$ of the cases, and none of these women were subsequently found to have greater than CIN 1 pathology. Researchers in this study referred to the fact that ECC results of less than CIN 1 pathology will spontaneously regress and therefore are inconsequential. Pretorius et. al [6] reported higher rates of positive ECC in ASCUS and LGSIL cytology compared to Williams and colleagues' (15.6\% and $14.8 \%$ with greater than or equal to CIN 2, respectively) ${ }^{[5]}$ but noted a much higher rate of positive ECCs in the setting of HGSIL on referral cytology ( $38 \%$ with greater than or equal to CIN2). This study had comparable results to Williams and colleagues, but additionally noted that most positive ECC results were in the setting of high grade cytology ${ }^{[6]}$.

\section{Opponents of ECC}

In some studies that were reviewed, researchers stated that ECC is not necessary. For example, Massad and Collins ${ }^{[7]}$ reported that a positive ECC was associated with serious lesions at follow-up; however, of all ECCs performed, only $1.4 \%$ were diagnosed with CIN 2 or CIN 3 based solely on ECC result. These investigators concluded that the use of ECCs failed to provide benefit in $94 \%$ of patients and considered it unnecessary in nulliparous women who had satisfactory colposcopic examination (recommendations regarding multiparous women were not specified in this study, although multiparous women were included in the analysis). Researchers in this study cited that only a small percentage (approximately 1.4\%) of patients would receive benefit from an ECC at the time of colposcopy because only CIN 2 and CIN 3 pathology of ECC was considered a beneficial finding (presumably to provide evidence for intervention). Irvin and colleagues ${ }^{[8]}$ in a study of 304 women reported that ECCs were positive in $6.4 \%$ of study cases but that the ECCs would have altered the evaluation plan in only $4.3 \%$ of women. Additionally, no occult cancer would have been missed if the ECCs were omitted and the only case of invasive cancer was found in a woman who had a negative ECC. This study showed that only $4.3 \%$ of women would have had their plan of care altered by performing ECC at the time of colposcopy and gives strength to the argument that even if ECC results are positive in a very small percentage of women, it does not significantly change their follow-up plan of care.

\section{False positive results and false negative results}

Anderson et al. ${ }^{[9]}$ reported that ECC results missed $45 \%$ of lesions in the endocervical canal that were identified on subsequent conization specimens and reported a $25 \%$ false-positive rate. Additionally, in a study conducted by el Dabh and colleagues ${ }^{[10]}$, ECC results were positive in 96 women who had a satisfactory colposcopic examination. The investigators measured the extent of the abnormality into the endocervical canal in the conization specimens; than or equal to $5 \mathrm{~mm}$ was considered a true-positive result relative to the ECC result. The false-positive rate was $82 \%$ and the investigators concluded that ECCs were not necessary. The previous studies indicate the conflicting issues related to ECCs at time of colposcopy related to false negative and false positive results and there have been false positive ECC results that could potentially lead to interventional management that is not necessary. Conversely, there have been findings that indicated that ECCs have missed lesions in the endocervical canal that were later identified on conization specimens.

\section{Contamination}

Spirtos and colleagues ${ }^{[11]}$ performed a study of 210 women who had satisfactory colposcopies and 51 women who had unsatisfactory colposcopies and then had colposcopies re-performed after the ECCs to determine if an ectocervical lesion was disrupted during the performance of the ECC. In those who had a satisfactory colposcopy, all those who had a positive ECC (9.5\%) had a disrupted ectocervical lesion. Of those women with unsatisfactory colposcopy in whom cold knife 
conization was performed ( 39 women), 4 (10\%) were diagnosed with squamous carcinoma. The authors suggested that if the ectocervical lesion is disrupted, the ECC could reflect the actual pathology of the ectocervix, not the endocervical canal. Additionally, they suggested that with an unsatisfactory colposcopy, ECC should be performed because of the possibility of occult disease. These investigators concluded that if an ECC was performed in a woman who had a satisfactory colposcopic examination, that colposcopy should be performed after the ECC, and if a lesion was disrupted, a more conservative follow-up as opposed to an excisional procedure for a positive ECC should be considered.

\section{Argument for doing ECC because of potential for missing serious lesions (and/or the ECC as the only manifestation of cervical disease)}

There was conflicting information in the literature related to the importance of ECC performance at time of colposcopy relative to missing pathology in the endocervical canal. Conflicting studies were as follows. Moniak et. al. ${ }^{[12]}$ correlated ECC results with disease locations on subsequently performed excisional specimens. In women whom the ECC was the only specimen positive for high -grade disease (CIN 2 or CIN 3), ectocervical lesions were present in $69 \%$, and $12.5 \%$ were high-grade endocervical lesions. Investigators therefore concluded that ECCs should be performed in all women because of the ability to detect lesions that otherwise might be missed by colposcopy and biopsy, although the investigators acknowledged that ECC is most likely to detect ectocervical disease. Soisson et. al. ${ }^{[13]}$ reported that in women who had positive ECCs, $9 \%$ had otherwise negative biopsies and another $9 \%$ had no other biopsy performed. In these situations, the majority of women $(80 \%)$ had dysplasia on the conization specimen, supporting the concept that ECCs should be performed as ECC was the only evidence of neoplastic epithelium. These investigators, however, did not distinguish low-grade from high-grade intraepithelial neoplasia on the conization specimen. Therefore, it cannot be determined whether or not the ECC was the only indication for subsequent evaluation leading to diagnosis of high-grade disease. In the study by Hatch et. al. ${ }^{[14]}$ of the 15 women diagnosed with squamous carcinoma, ECC was the only evidence of invasion in 7 of these women (47\%), although the investigators noted that the ECC was useful mostly in detecting disease in women who had an unsatisfactory or normal colposcopic examination. Fine et al. ${ }^{[15]}$ reported that 6 of 17 women diagnosed with carcinoma and a positive ECC had normal colposcopy and in 5 or 6 of these women, positive ECCs were the only indication to perform a cone biopsy. Ferenczy ${ }^{[16]}$ reported that 19 women who had unsuspected carcinoma were identified with ECCs, although 18 of these 19 women also had unsatisfactory colposcopy.

\section{Using ECC as a predictive value for micro invasion}

A study by Helmerhorst ${ }^{[17]}$ indicated the positive and negative predictive values of ECC in women who had satisfactory colposcopy were $2.4 \%$ and $99.4 \%$. For women with unsatisfactory colposcopies, the positive and negative predictive values were $22.4 \%$ and $96.7 \%$. The negative predictive values were similar regardless of the adequacy of the colposcopic examination. The conclusion of this study was that the impact of ECCs was limited; in those who had satisfactory colposcopy, ECCs may have reduced under diagnosis. In women who had unsatisfactory colposcopy, however, the predictive value for invasive disease was increased.

\section{Is ECC necessary in patients with CIN 1 and adequate colposcopic examination?}

A study by Naumann et al. ${ }^{[18]}$ had particular significance to the proposed research study because the question was raised whether it was necessary to perform ECC in patients with CIN 1 cytology; however, this study did not include women with HRHPV or note the age of study participants. Naumann stated, "Our study suggests that ECC is not necessary for patients with an adequate colposcopic examination when referral Pap smears show CIN 1. We found that the rate of dysplasia on ECC in patients referred with CIN 1 was less than $1 \%$ and the only ECC specimen positive for atypia contained mild dysplasia. It is also likely that this positive ECC result was due to ectocervical contamination. Further, the clinical significance of CIN 1 in the endocervical canal is debatable, since untreated CIN 1 has been shown to have a relatively benign natural history and a high likelihood of spontaneous regression". (P. \# 968). 


\section{Is it worth performing ECCs in women less than 35 years old?}

There was an absence in the literature about performing ECCs in women 35 years of age or less. There were two age related studies in the literature related to performing ECC at the time of colposcopy and they involved women less than 40 years of age and women who were 35 to 45 years of age. In the ASCUS/LSIL Triage Study (ALTS) trial, it was found that the sensitivity of biopsy and the sensitivity of ECC for cumulative CIN 2+ varied by age: biopsies were more sensitive in younger than in older women, whereas the reverse was found for ECC ${ }^{[1]}$. Therefore, the marginal contribution of ECC (i.e., in addition to the biopsy) was accentuated in older women. In women less than 40 years of age, ECCs yielded only $2 \%$ increased detection of CIN 2+ over the biopsy findings. By contrast, in women 40 years of age and older, ECCs increased sensitivity for CIN 2+ by 13 Researchers noted that while performing an additional test will always increase sensitivity, the marginal gain in sensitivity must be balanced against the costs in terms of patient discomfort and the costs of testing ${ }^{[1]}$. Furthermore, the study showed that among 888 women under the age 40, biopsy detected CIN $2+$ in 235 and of the remaining 653 women; seven cases of CIN 2+ were detected by ECCs alone. Therefore, the risk of undetected CIN $2+$ associated with not performing ECCs would have been 7 of 653 , or $1.1 \%{ }^{[1]}$.

Li, Zhang, Wu, Li A., Qiao, Li L, and Pan ${ }^{[19]}$ reported in the Chinese Journal of Oncology a study of women who were approximately 35 to 45 years of age. Among the 1997 women who received ECC, 31 were positive for abnormal histological changes with a frequency of 1.6\%. Of these 31 cases, 9 had LGSIL (0.5\%), 20 had HGSIL (1.0\%) and 2 had squamous-cell carcinoma. No diagnosis of pathology could be made for 131 women because of insufficient tissue. Women with positive cytological findings had a higher frequency (5.3\%) of abnormal ECC than those with negative cytological findings $(0.3 \%)$ and there was positive correlation between the frequency of abnormal ECC and the grade of cytological findings. It was found that abnormal ECCs were present in $9.1 \%$ of those with unsatisfactory colposcopy, compared to $1.3 \%$ of those with satisfactory colposcopy. Frequency of abnormal ECC was $0.6 \%$ in patients with negative colposcopy, $0.9 \%$ in LGSIL and $24.1 \%$ in HGSIL. Positive rate of ECC was 3.3\% in LGSIL, 22.2\% in HGSIL and 50.0\% in squamous carcinoma. Of 316 patients with positive cytology but negative colposcopy, the ECC was abnormal in 8 patients $(2.5 \%)$ and 3 HGSILs were histologically verified. The authors concluded that if cytology or colposcopy shows HGSIL or more severe changes, and cytology is positive while colposcopy is unsatisfactory, ECCs should be done routinely.

\section{Marginal diagnostic yield}

The results of the following study suggested that positive ECC could be related to positive ectocervical pathology that had contaminated the ECC sample; this is an idea that was previously discussed in the contamination section.

In the ALTS trial, overall, $3 \%$ to $4 \%$ of ECC samples were diagnosed as CIN2+; the marginal diagnostic yield (independently of biopsy) was only $0.9 \%$. When CIN $2+$ was diagnosed only by ECC, and not by biopsy, women were less likely to have a visible acetowhite lesion, suggesting the lesion was located in the canal. However, in one third of women with concordantly positive biopsies and ECC, an acetowhite lesion was identified close to the os. This raised the possibility that the positive ECC was due to tissue contamination in some of the cases and emphasized the importance of the Colposcopists taking care to avoid contamination of ECC specimens when lesions are located near the os ${ }^{[1]}$.

\section{Diagnostic utility}

The diagnostic utility of ECCs has been questioned in several studies. A study by Irvin and colleagues ${ }^{[8]}$ reported low diagnostic utility of ECC of 304 patients that were evaluated because of abnormal cytology, the results of ECCs changed the management of 13 patients $(4.3 \%)$. None of these patients had an underlying carcinoma that would have been missed if ECCs had not been performed. The authors concluded that ECCs did not significantly contribute to the management of women with abnormal cytology. They also stated that performance of ECCs did not help to avert conization in women with unsatisfactory colposcopy because they believed that a negative ECC cannot be relied upon to rule out endocervical 
disease or invasion (in such cases). Instead, they advocated the performance of conization on all women with abnormal high-grade cytology and unsatisfactory colposcopy.

Ferenczy ${ }^{[16]}$ advocated the use of ECCs along with colposcopic evaluations in all women being evaluated for abnormal cytology. He argued that a negative ECC would help to avoid diagnostic cold knife conization for women presumably without disease in the endocervical canal. Conversely, women with positive ECCs, (implying endocervical lesions), could be sent directly to cold knife conization or a more definitive surgical procedure without further outpatient treatment. Ferenczy emphasized that the utility of ECCs is contingent upon accurate sampling of endocervical cells, preparation of the specimen, and pathologic interpretation and that ECC specimens are often misinterpreted due to abundant blood, mucus and other cellular material that obscure the epithelium.

Massad and Collins ${ }^{[7]}$ studied the predictors of an abnormal ECC to determine what factors increase the yield of this procedure in the workup of patients with abnormal cytology. Among 2,287 women undergoing colposcopies, an abnormal ECC changed the management of 105 women (4.6\%). They found that older women, with higher parity, earlier age at first intercourse, and an unsatisfactory colposcopy were more likely to have an abnormal ECC result. They concluded that ECC may be avoided in women with satisfactory colposcopy who have no colposcopic lesions.

Pretorius and colleagues ${ }^{[6]}$ recently studied 364 women from a screening population in China who had satisfactory colposcopy and CIN 2+ final pathologic results and found that 20 of the 364 cases (5.5\%) of CIN 2+ were diagnosed solely by an ECC result. They concluded that ECCs should be performed, even when the colposcopic examination is satisfactory. However, it is important to note that the women in this study were older as compared with ALTS, with a mean age of 42 years of age (range 32 to 50 years of age). Solomon et al. ${ }^{[1]}$ stated after an analysis of data, "In our analysis, ECC showed minimal diagnostic utility in women under 40, suggesting this common procedure may be overused. Although ECC did increase sensitivity for detection of CIN $2+$ among women 40 and over, conclusions must be tempered by the relatively small number of cases in this analysis”. (p. \#295)

\section{Gaps in the literature related to ECC}

The review of literature revealed gaps in the literature relative to ECC, that is, there were no age based studies (of women less than 35 years of age), no studies based on patients with LGSIL or HRHPV cytology, no randomized studies, and no systematic reviews of literature addressing ECCs in the evaluation of squamous abnormalities. For example, studies in which researchers clearly illuminate gaps in knowledge were further expanded upon in the ALTS trial. It was observed that : (a) ECCs were performed in 1,524 (18.4\%) of 8,265 total colposcopy procedures, including multiple colposcopies for many women, (b) ECCs were performed at the clinician's discretion, and clinicians varied in their frequency of obtaining an ECC, (c) Women who were older were more likely to have an ECC performed than were younger women, (d) There were clinical center-specific tendencies in the use of ECC, even adjusted for all other variables and, (e) Not surprisingly, there was a significant trend with increasingly severe enrollment cytological abnormality: women with HGSIL were more than five times more likely to have an ECC performed than women with negative cytology ${ }^{[1]}$. ALT's was not a study designed to evaluate endocervical sampling. ECCs were performed inconsistently and therefore only data on a subset of women were available, true sensitivity and specificity cannot be provided with these ECC results. Another caveat is that women in ALTS were referred with minor cytological abnormalities and were followed intensively. Therefore, CIN 2+ lesions identified in ALTS were probably, on average, smaller and less severe compared with lesions diagnosed in general practice. In addition, for analyses of cumulative CIN $2+$ some cases detected in follow-up or at exit may have been incident (not missed prevalent) disease ${ }^{[1]}$. An additional point was that the clinical significance of CIN 1 in the endocervical canal is debatable since untreated CIN 1 has been shown to have a relatively benign natural history and a high likelihood of spontaneous regression. 


\section{Conclusions}

The literature review provides a critique of the studies that were reviewed, an analysis of the literature, details gaps in the literature and enumerates the problem of no evidence based guidelines for ECC at the time of colposcopy in women who are less than 35 years of age with LGSIL or HRHPV. The available evidence about the need for ECC at the time of colposcopy in women who are less than 35 years of age with Cervical Cytology of LGSIL or HRHPV at the time of colposcopy is inconclusive, and there does not appear to be a general consensus. Specifically, there are no studies in which researchers evaluate the effectiveness or utility of ECC in patients less than or equal to 35 years of age. The literature review that was conducted establishes a need for a study that begins to establish evidence based guidelines for ECC that could be utilized in practice in this particular group of women.

\section{I mplications for future study}

The literature review establishes the foundation and background for future studies that examine the need for ECC. Several future studies are indicated based on the results of the literature review. Additional studies could include variables such as race, tobacco use, age at first pregnancy, age at first intercourse, smoking history, parity, previous treatment for CIN, previous diagnoses of cervical pathology, and women who are $\geq 35$ years of age, to increase the number and variation of subjects that meet study criteria and ultimately enhance the generalizability of the study.

\section{References}

[1] Solomon, D., Stoler, M., Jeronimo, J., Khan, M., Castle, P., Schiffman, M. Diagnostic utility of endocervical curettage in women undergoing colposcopy for equivocal or low-grade cytologic abnormalities. Obstetrics and Gynecology. 2007; $110(2)$ : $288-297$. http://dx.doi.org/10.1097/01.AOG.0000270154.69879.09

[2] Auston, I., Cahn, M.A., Seldon, C. R. Literature search methods for the development of clinical practice Guidelines. National Library of Medicine, Office of Health Services Research Information [Internet]. Retrieved November 30, 2011. Available from: http://www.nlm.nih.gov.nichsr/litsrch.htm

[3] Krebs HB, Wheelock JB, Hurt WG. Positive endocervical curettage in patients with satisfactory and unsatisfactory colposcopy: clinical implications. Obstet Gynecol 1987; 69(4):601-5. PMid:3822303

[4] Saltzman, D. H., Evans, M. I., Warsof, S. L., et al. Endocervical curettage as a routine part of colposcopic examinations of abnormal cervical cytology. Journal of Reproductive Medicine. 1985; 30(11): 871-873. PMid:4078822

[5] Williams, D. L., Dietrich C., McBroom J. Endocervical curettage when colposcopic examination is satisfactory and normal. Obstetrics and Gynecology. 2000; 95(6): 801-803. http://dx.doi.org/10.1016/S0029-7844(00)00821-8

[6] Pretorius, R. J., Zhang, W. H., Belinson, J. L., et al. Colposcopically directed biopsy, random cervical biopsy, and endocervical curettage in the diagnosis of cervical intraepithelial neoplasia 2 or worse. American Journal of Obstetric Gynecology. 2004; 191(2): 430-434. PMid:15343217 http://dx.doi.org/10.1016/j.ajog.2004.02.065

[7] Massad, L. S., Collins, Y. C. Using history and colposcopy to select women for endocervical curettage. Results from 2,287 cases. Journal of Reproductive Medicine. 2003; 48(1): 1-6. PMid:12611087

[8] Irvin, W., Flora, S., Anderson, W., et al. Endocervical curettage. Does it contribute to the management of patients with abnormal cervical cytology? Journal of Reproductive Medicine. 2004; 49(1): 1-7. PMid:14976787

[9] Anderson, W., Frierson, H., Barber, S., Tabbarah, S., Taylor, P., Underwood, P. Sensitivity and specificity of endocervical curettage and the endocervical brush for the evaluation of the endocervical canal. American Journal of Obstetrics and Gynecology. 1988; 159(3): 702-704. PMid:3048105

[10] Dabh, A., Rogers, R.E., Davis, T.E., Sutton, G. The role of endocervical curettage in satisfactory colposcopy. Obstetrics and Gynecology. 1989; 74(2): 159-164. PMid:2748050

[11] Spirtos, N.M., Schlaerth, J.B., dAbling, G., 111, \& Marrow, C. P. A critical evaluation of the endocervical curettage. Obstetrics and Gynecology. 1987; 70(5): 729-733. PMid:3658281

[12] Moniak CW, Kutzner S, Adam E, et al. Endocervical curettage in evaluating abnormal cervical cytology. J Reprod Med. 2000; 45(4): 285-92. PMid:10804483

[13] Soisson A.P., Molina C.Y. Benson W.L. Endocervical curettage in the evaluation of cervical disease in patients with adequate colposcopy. Obstet Gynecol. 1988; 71(1): 109-111. PMid:3336531

Published by Sciedu Press 
[14] Hatch KD, Shingleton HM, Orr JW Jr, et al. Role of endocervical curettage in colposcopy. Obstet Gynecol. 1985; 65(3):403-8. PMid:3974966

[15] Fine BA, Feinstein GI, Sabella V. The pre- and postoperative value of endocervical curettage in the detection of cervical intraepithelial neoplasia and invasive cervical cancer. Gynecol Oncol 1998; 71(1):46-9. PMid:9784317 http://dx.doi.org/10.1006/gyno.1998.5120

[16] Ferenczy, A. Endocervical curettage has no place in the routine management of women with cervical intraepithelial neoplasia: Debate. Clinical Obstetrics and Gynecology. 1995; 38(3): 644-648. PMid:8612374 http://dx.doi.org/10.1097/00003081-199509000-00024

[17] Helmerhorst TJ. Clinical significance of endocervical curettage as part of colposcopic evaluation. A review. Int J Gynecol Cancer 1992; 2(5): 256-62. PMid:11576267 http://dx.doi.org/10.1046/j.1525-1438.1992.02050256.x

[18] Naumann, R. W., Crispens, M. A., Alvarez, R. D., Patridge, E., Shingelton, H., \& Kilgore, L. Treatment of cervical dysplasia with large loop excision of the transformation zone: is endocervical curettage necessary? Southern Medical Journal. 1996; 89(10): 961-965. PMid:8865787 http://dx.doi.org/10.1097/00007611-199610000-00005

[19] Li, N., Zhang, W.H., Wu, L. Y, Li, A. L., Qiao, Y. L., Li, L., et al. Chinese cervical cancer pilot study. Chinese Journal of Oncology. 2004; 26(7): 406-408. PMid:15355644 